\title{
Óleo essencial da Canela (Cinamaldeído) e suas aplicações biológicas
}

\author{
Cinnamon essential oil (cinnamaldehyde) and its applications
}

Cristiane Santos Silva e Silva Figueiredo ${ }^{1}$, Patrícia Viera de Oliveira ${ }^{2}$, Warlison Felipe de Silva Saminez ${ }^{3}$, Roseana Muniz Diniz ${ }^{4}$, João Francisco Silva Rodrigues ${ }^{5}$, Mari Silma Maia da Silva ${ }^{6}$, Luís Cláudio Nascimento da Silva ${ }^{7}$, Marcos Augusto Grigolin Grisotto ${ }^{8}$.

Resumo: Cinamaldeído (CND) é o principal componente ativo do óleo essencial da canela (Cinnamomum sp) e tem sido amplamente utilizado em atividades biológicas e farmacológicas, tendo sido relatado atividade antimicrobiana, antioxidante, antidiabética, dentre outras. Devido os diversos relatos das propriedades farmacológicas do composto, esse foi escolhido para revisão de literatura. A seleção da bibliografia foi obtida a partir de bases de dados (google scholar, NCBI - National Center for Biotechnology Information, PubMed e Scielo - Scientific Electronic Library Online). A composição química do CND tem compostos terpenóides que têm poderosa atividade antimicrobiana contra fungos, bactérias Gram-positivas e Gram-negativas tais como Aeromonas hydrophila, Enterococcus faecalis, Clostridium botulinum, Staphylococcus aureus, Escherichia coli O157: H7 e Salmonella enterica serovar Typhimurium. A atividade anti-inflamatória induz a apoptose e inibi a proliferação celular, nas respostas imunes mediadas por monócitos/macrófagos, além de diminuir a produção de óxido nítrico induzido por lipopolissacáridos de um modo dependente da dose. CND ao ser administrado por via oral em ratos diabéticos demonstrou melhora no conteúdo de glicogênio muscular e hepático aumentando a liberação de insulina. Além disso CND estimulou a angiogênese in vivo e in vitro, regulando positivamente o fator de crescimento endotelial vascular. A partir do que foi relatado constatou-se que o CND possui muitas atividades com potencial farmacológico, mas percebe-se que se faz necessário estudos sobre o(s) mecanismos(s) de ação dessas atividades a fim de se proporcionar o uso seguro e eficaz do cinamaldeído.

Palavras-chave: Cinnamomum sp, cinamaldeído, atividades biológicas.

\begin{abstract}
Cinnamaldehyde (CND) is the main active component of cinnamon essential oil (Cinnamomum sp) and has been widely used in biological and pharmacological activities, and antimicrobial, antioxidant and antidiabetic activities have been reported. Due to the diverse reports of the pharmacological properties of the compound, it was chosen for literature review. The selection of the bibliography was obtained from databases (google scholar, NCBI - National Center for Biotechnology Information, PubMed and Scielo - Scientific Electronic Library Online). The chemical composition of CND has terpenoid compounds that have potent antimicrobial activity against fungi, Gram-positive and Gram-negative bacteria such as Aeromonas hydrophila, Enterococcus faecalis, Clostridium botulinum, Staphylococcus aureus, Escherichia coli 0157: H7 and Salmonella enterica serovar Typhimurium. The anti-inflammatory activity induces apoptosis and inhibits cell proliferation in monocyte/macrophage mediated immune responses and decreases the production of nitric oxide induced by lipopolysaccharides in a dose-dependent manner. CND when administered orally in diabetic rats demonstrated an improvement in the muscle and liver glycogen content, increasing the release of insulin. In addition, CND stimulated angiogenesis in vivo and in vitro, regulating vascular endothelial growth factor positively. Based on what has been reported, it has been observed that CND has many activities with pharmacological potential, but it is perceived that it is necessary to study the mechanism (s) of action of these activities in order to provide the use safe and effective use of cinnamaldehyde.
\end{abstract}

Key words: Cinnamomum sp, cinnamaldehyde, biological activities.

\footnotetext{
${ }^{1,5}$ Alunos de Pós-graduação do Programa de Doutorado da Rede de Biodiversidade e Biotecnologia da Amazônia Legal - BIONORTE. Universidade Ceuma.

${ }^{2,3,4}$ Alunos de Graduação do curso de Biomedicina da Universidade Ceuma.

${ }^{6}$ Aluna de Pós-graduação do Programa de Mestrado em Biologia Parasitária da Universidade Ceuma.

7,8 Docentes da Universidade Ceuma.
}

\section{Autor correspondente:}

Cristiane Santos Silva e Silva Figueiredo.e-mail: cristianeloud@gmail.com

Endereço: Rua Projetada, Residencial Marcelle I, Turu Telefone: (98) 981484299

Rev. Investig, Bioméd. São Luís, 9(2): 192-197, 2017 


\section{Introdução}

A canela (Cinnamomum sp) tem sido amplamente utilizada como especiaria, bem como na medicina tradicional à base de ervas em todo o mundo e tem uma longa história de uso como tempero e agentes aromatizantes. Ela é tradicionalmente colhida em países asiáticos e amplamente cultivada em Madagascar, no Sri Lanka, na Indonésia e no sul da China ${ }^{1}$. Os constituintes químicos mais importantes da canela são os óleos voláteis (cinamaldeído, eugenol e ácido cinâmico), mucilagem, diterpenos e proantocianidinas $^{2}$.

O cinamaldeído (aldeído cinâmico ou 3-fenil-2-propenal), um álcool terpeno cíclico, é o principal componente ativo do óleo essencial de canela $(60-75 \%)$. É aprovado pela Food and Drug Administration (FDA) e tem sido amplamente utilizado em goma, sorvete, doces, bebidas, pães, cereais $^{3}$ e $\operatorname{cosméticos}^{4}$. No entanto, a aplicação do cinamaldeído (CND) na conservação de alimentos é limitada pelo seu sabor particular, volatilidade e natureza lipofílica

Os óleos possuem várias atividades biológicas, incluindo atividade antimicrobiana de amplo espectro, antioxidantes e antiapoptótico ${ }^{6,7,8}$. Além disso possuem efeitos antidiabéticos ${ }^{9}$, anticancerígenos $^{10}$, antitumoral $^{11,12} \mathrm{e}$ inibem a neuroinflamação ${ }^{13}$.

Possui ainda atividade cicatrizante ${ }^{14}$, de mediador de monócitos e macrófagos ${ }^{15}$, anticancro e 0 óleo essencial, cinamaldeído, foi considerado pouco tóxico para os seres humanos ${ }^{6}$. A casca de Cinnamomum cassia foi aprovada como medicamento fitoterápico pela Farmacopeia Chinesa como analgésico e para promover a circulação sanguínea ${ }^{16}$.

Devido aos diversos relatos das propriedades farmacológicas do cinamaldeído, principal constituinte do óleo essencial da canela, o mesmo foi escolhido para a revisão de literatura. $O$ presente artigo de revisão tem por objetivo relatar as principais atividades biológicas do óleo essencial da Cinnamomum sp. A seleção de fatos, informações e bibliografia foram obtidos das principais bases de dados de literatura tais como: google scholar, NCBI (National Center for Biotechnology Information), PubMed e Scielo (Scientific Electronic Library Online).

\section{Discussão}

\section{Atividade Antimicrobiana}

Tem sido relatado que 0 cinamaldeído, que contem compostos terpenóides ou fenólicos, hidrofóbicos, têm poderosa atividade antimicrobiana contra uma variedade de patógenos ${ }^{17}$, incluindo fungos, bactérias Gram-positivas e Gramnegativas ${ }^{18}$.

Sanla-Ead, e colaboradores $^{19}$ obtiveram boa atividade antibacteriana, a partir de filmes a base de celulose adicionado ao CND, com concentrações inibitórias mínimas que variaram de 0,78 a $50 \mu \mathrm{l} / \mathrm{ml}$ contra as bactérias Aeromonas hydrophila e Enterococcus faecalis.

Gill e Holley ${ }^{14}$ concluíram que a Concentração Inibitória Mínima (CIM) de CND contra Listeria Monocytogenes é 40 $\mathrm{mM}$ e ainda foram mais além ao determinar a via de inibição. Ao utilizar cianeto de carbonilo m-clorofenil-hidrazona (CCCP) e CND durante 5 minutos observaram o declínio no aumento da Concentração de ATP após adição de glicose inibindo o crescimento celular.

Os efeitos sobre a geração de energia desempenham claramente um papel importante na atividade do cinamaldeído em concentrações bactericidas. Os possíveis mecanismos de inibição da geração de energia são a inibição de captação de glicose ou 
utilização de glicose e efeitos sobre a permeabilidade da membrana ${ }^{13}$.

Foi relatado também que o cinamaldeído inibe 0 crescimento de Clostridium botulinum, Staphylococcus aureus, Escherichia coli O157: H7 e Salmonella enterica serovar Typhimurium $^{20}$. CND ao ser encapsulado em quitosana ou em nano fibras de polivinil, mostrou excelente atividade antimicrobiana contra Escherichia coli, Pseudomonas aeruginosa e Staphylococcus aureus ${ }^{21,17}$.

Em testes realizados com Porphyromonas gingivalis, o CND inibiu o crescimento na CIM de 2,5 $\mu \mathrm{M}$. Foi observado ainda que houve vazamento de ácido nucléico e proteína em concentrações crescentes de cinamaldeído. Verificou-se dano a integridade da membrana de $P$. gingivalis aumentando a permeabilidade celular e alterações que indicou destruição da membrana celular. Para determinar ainda mais o efeito anti-biofilme, examinou-se a formação relativa de biofilme e biofilmes estabelecidos, demonstrando que cinamaldeído a níveis sub-CIM inibiram a formação de biofilme de $P$. gingivalis em $74,5 \%$ e $67,3 \%$ separadamente o que sugere o potencial do cinamaldeído como um agente antimicrobiano natural contra a doença periodontal ${ }^{22}$.

\section{Atividade Anti-inflamatória e Respostas Imunes}

Cinamaldeído induz a apoptose $e^{23}$ e inibe a proliferação celular ${ }^{24}$, nas respostas imunes mediadas por monócitos/macrófagos. Kim; Lee e colaboradore $^{25}$ utilizaram macrófagos ativados por lipopolissacarídeo (LPS) e monócitos tratados com anticorpos próagregativos e proteína de matriz extracelular, fibrobectina. O CND foi capaz de suprimir tanto a produção de óxido nítrico (NO) quanto a regulação positiva dos níveis superficiais de moléculas coestimulatórias (CD80 e CD69) e receptores de reconhecimento de padrões (receptor toll-like 2 (TLR2) e receptor de complemento (CR3)).

Além disso, o óleo também bloqueou a adesão célula-célula induzida pela ativação de CD29 e CD43. A análise de imunoblotting sugeriu que a ação do cinamaldeído foi devida à inibição da fosfoinositide-3-quinase (PI3K) e quinase dependente de fosfoinositídeos (PDK) 1, bem como à ativação do fator nuclear (NF $\mathrm{KB})^{24}$. Os resultados descritos sugerem que o cinamaldeído pode atuar como um forte regulador de respostas imunes mediadas por monócitos/macrófagos.

Foi investigado os efeitos antiinflamatórios do transcinamaldeído na linhagem celular de macrófagos murinos RAW 264.7. Houve diminuição da produção de óxido nítrico (NO) induzida por lipopolissacáridos (LPS) de um modo dependente da dose, além da redução da expressão de mRNA e a expressão proteica da NO sintase indutível (iNOS) em macrófagos estimulados por LPS. O tratamento também diminuiu o nível de expressão de mRNA e a secreção de IL$1 \beta$, IL-6 e TNF- $\alpha$ em macrófagos ativados por LPS, inibindo a fosforilação de ERK, JNK e p38 MPAK nas células ${ }^{25}$ demonstrando que 0 trancinamaldeído pode tem potencial farmacológico para inibir a inflação.

\section{Outras Atividades Relatadas}

O diabetes mellitus é um distúrbio metabólico crônico que afeta cerca de $6 \%$ da população mundial com suas complicações e está rapidamente atingindo a escala epidêmica. Cinnamomum 
zeylanicum é amplamente utilizado em sistema alternativo de medicina para tratamento de diabetes ${ }^{27}$.

Os pesquisadores administraram por via oral o cinamaldeído $(20 \mathrm{mg} / \mathrm{kg})$ em ratos diabéticos por 2 meses, demosntrando melhora significativa ( $p$ $<0,001)$ no conteúdo de glicogênio muscular e hepático. A incubação in vitro de ilhotas pancreáticas com cinamaldeído aumentou a liberação de insulina em comparação com a glibenclamida. Verificou-se que o efeito insulinotrópico do óleo aumenta a captação de glicose através da translocação do transportador de glicose (GLUT4) nos tecidos periféricos. O tratamento também mostrou melhora significativa nas atividades enzimáticas alteradas de piruvato quinase (PK) e fosfoenolpiruvato carboxiquinase (PEPCK) e seus níveis de expressão de mRNA. Além disso, a dose letal mediana $\left(\mathrm{DL}_{50}\right)$ de cinamaldeído não pôde ser obtida nem mesmo 20 vezes $(0,4 \mathrm{~g} / \mathrm{kg})$ de sua dose efetiva $^{27}$. Com a alta margem de segurança utilizada o cinamaldeído pode ser desenvolvido como um potencial terapêutico candidato para o tratamento da diabetes.

Cinamaldeído também estimulou estimula a angiogênese in vivo e in vitro, regulando positivamente 0 fator de crescimento endotelial vascular (VEGF) e a expressão de $\mathrm{Flk}-1 / \mathrm{KDR}^{28}$. No seguinte estudo, verificou-se que o CND tem propriedades pró-angiogênicas contra as células endoteliais da veia umbilical humana (HUVECs). Demonstrou-se que nas HUVECs, o CND estimulou a proliferação, migração, formação de tubos, ativou as vias da fosfatidilinositol 3-quinase (PI3K) e da proteína quinase mitogênica (MAPK). Além disso, a secreção do fator de crescimento endotelial vascular (VEGF) das HUVECs foi aumentada. In vivo, o óleo restaurou parcialmente os vasos intersegmentares no peixe-zebra pré- tratado com PTK787, um inibidor seletivo do receptor do fator de crescimento endotelial vascular (VEGFR), mostrando eficácia pró-angiogênica ${ }^{29}$.

Além disso, CND atenuou tamanhos de ferida em modelo de ferida cutânea, e proteína VEGF elevada e densidade vascular CD31-positiva na margem dessas feridas. Estes resultados ilustram que o CND acelera a cicatrização de feridas induzindo a angiogênese na área da ferida. O mecanismo potencial envolve a ativação das vias de sinalização PI3K / AKT e $M{ }^{2} K^{28}$. Então CND, molécula não peptídica, pode ter aplicações clínicas para promover a angiogênese terapêutica em feridas diabéticas crônicas e enfarte do miocárdio.

\section{Conclusão}

A partir dos trabalhos expostos constatou-se que o Cinamaldeído possui muitas atividades com potencial farmacológico que contemplam atividade antimicrobiana, anti-inflamatória, angiogênica, cicatrizante, dentre outras, além de ser considerado de baixa toxicidade, mas percebe-se que se faz necessário estudos sobre $\mathrm{O}(\mathrm{s})$ mecanismos(s) de ação dessas atividades a fim de se proporcionar o uso seguro do cinamaldeído. 


\section{Referências}

1. Burt, S. Essential oils: their antibacterial properties and potential applications in food - a review. International Journal of Food Microbiology 2004; 94: 223-253.

2. Jayaprakasha, G. K.; Raom, L. J.; Sakariah, K. $\mathrm{K}$. Chemical composition of volatile oil from Cinnamomum zeylanicum buds. Z. Naturforsch. 57(12):990-993, 2002.

3. López-Mata, M.A.; Ruiz-Cruz, S.; de Jesús Ornelas-Paz, J.; del Toro-Sánchez, C.L.; Márquez-Ríos, E.; Silva-Beltrán, N.P.; CiraChávez, L.A.; Burruel-Ibarra, S.E. Mechanical, barrier and antioxidant properties of chitosan films incorporating cinnamaldehyde. J. Polym. Environ. 1-10, 2017.

4. Lee, R.; Balick, M.J. Sweet wood-cinnamon and its importance as a spice and medicine. J. Sci. Heal. 1, 61-64, 2005.

5. Amara, A. A.; El-Masry, M. H.; Bogdady, H. H. Plant crude extracts could be the solution: Extracts showing in vivo antitumorigenic activity. Pak. J. Pharm. Sci. 21:159-171, 2008.

6. Shen, S.; Zhang, T.; Yuan, Y.; Lin, S.; Xu, J.; Ye, $\mathrm{H}$. Effects of cinnamaldehyde on Escherichia coli and Staphylococcus aureus membrane. Food Control. 47, 196-202, 2015. doi: 10.1016/j.foodcont.2014.07.003

7. Chen, W.; Golden, D. A.; Critzer, F. J.; Davidson, P. M. Antimicrobial activity of cinnamaldehyde, carvacrol, and lauric arginate against Salmonella Tennessee in a glycerol-sucrose model and peanut paste at different fat concentrations. J. Food Prot. 78, 1488-1495, 2015. doi: 10.4315/0362-028X.JFP14-599.

8. Utchariyakiat, I.; Surassmo, S.; Jaturanpinyo, M.; Khuntayaporn, P.; and Chomnawang, M. T. Efficacy of cinnamon bark oil and cinnamaldehyde on anti-multidrug resistant Pseudomonas aeruginosa and the synergistic effects in combination with other antimicrobial agents. BMC Complement. Altern. Med. 16:158. 2016. doi: 10.1186/s12906-016-1134-9

9. Sartorius, T.; Peter, A.; Schulz, N.; Drescher, A.; Bergheim, I.; Machann, J.; Schick, F.; SiegelAxel, D.; Schürmann, A.; Weigert, C.; et al. Cinnamon extract improves insulin sensitivity in the brain and lowers liver fat in mouse models of obesity. PLoS ONE, 2014.

10. Kwon, H.K.; Hwang, J.S.; So, J.S.; Lee, C.G.; Sahoo, A.; Ryu, J.H.; Jeon, W.K.; Ko, B.S.; Im,
C.R.; Lee, S.J.; et al. Cinnamon extract induces tumor cell death through inhibition of NF_B and AP1. BMC Cancer, 10, 392-402, 2010.

11. Schoene, N.W., Kelly, M.A., Polansky, M.M., Anderson, R.A., Water-soluble polymeric polyphenols from cinnamon inhibit proliferation and alter cell cycle distribution patterns of hematologic tumor cell lines. Cancer Lett., 230: 134-140. 2005

12. Singh, Rana P.; Raina, Komal; Deep, Gagan; Chan, Daniel; Agarwal, Rajesh. Silibinin Suppresses Growth of Human Prostate Carcinoma PC-3 Orthotopic Xenograft via Activation of Extracellular Signal-Regulated Kinase 1/2 and Inhibition of Signal Transducers and Activators of Transcription Signaling. Clin Cancer Res January 15 (2): 613-621. 2009.

13. Ho, S.C.; Chang, K.S.; Chang, P.W. Inhibition of neuroinflammation by cinnamon and its main components. Food Chem. 138, 2275-2282, 2013.

14. Gill, A. O.; Holley, R. A. Mechanisms of Bactericidal Action of Cinnamaldehyde against Listeria monocytogenes and of Eugenol against L. monocytogenes and Lactobacillus sakei. Applied and Environmental Microbiology, oct. 2004, p. 5750-5755.

15. Cheng, A.G., McAdow, M., Kim, H.K., Bae, T., Missiakas, D.M., Schneewind, O. Contribution of coagulases towards Staphylococcus aureus disease and protective immunity. PLoS Pathog. 6, e1001036, 2010.

16. Commission CP. Chinese pharmacopoeia. Vol. 328. Beijing: Chemical Industry Press; 2005. p. 547. Packaging Technology and Science, 25: 71, 2012.

17. Rieger, K.A.; Schiffman, J.D. Electrospinning an essential oil: Cinnamaldehyde enhances the antimicrobial efficacy of chitosan/poly (ethylene oxide) nanofibers. Carbohydr. Polym. 113, 561568, 2014.

18. Chen, H.; Hu, X.; Chen, E.; Wu, S.; McClements, D.J.; Liu, S.; Li, B.; Li, Y. Preparation, characterization, and properties of chitosan films with cinnamaldehyde nanoemulsions. Food Hydrocoll. 61, 662-671, 2016.

19. Sanla-Ead, N.; Jangchud, A. Chonhenchob, P. S. V. Antimicrobial Activity of Cinnamaldehyde and Eugenol and Their Activity after Incorporation into Cellulose-based Packaging Films. 2012 
20. Helander, I. M.; Alakomi, H. L.; Latva-Kala, K.; Mattila-Sandholm, T.; Smid, E. J.; Gorris, L. G. M.; Wright, A. V. Characterization of the action of selected essential oil components on Gramnegative bacteria. J. Agric. Food Chem. 46:3590-3595, 1998.

21. Wen, P.; Zhu, D.-H.; Wu, H.; Zong, M.-H.; Jing, Y.-R.; Han, S.-Y. Encapsulation of cinnamon essential oil in electrospun nanofibrous film for active food packaging. Food Control. 59, 366376, 2016.

22. Wang, Y.; Zhang, Y. Shi, Y.; Pan, X.; Lu, Ping, $Y$. Antibacterial effects of cinnamon (Cinnamomum zeylanicum) bark essential oil on Porphyromonas gingivalis. Cao.Microbial Pathogenesis, 2018

23. Cabello, C.M.; Bair, W.B.; Lamore, S.D.; Ley, S.; Bause, A.S.; Azimian, S.; Wondrak, G.T. The cinnamon-derived Michael acceptor cinnamic aldehyde impairs melanoma cell proliferation, invasiveness, and tumor growth. Free Radic. Biol. Med., 46, 220-231. 2009.

24. Chuang, L.Y.; Guh, J.Y.; Chao, L.K.; Lu, Y.C.; Hwang, J.Y.; Yang, Y.L.; Cheng, T.H.; Yang, W.Y.; Chien, Y.J.; Huang, J.S. Anti-proliferative effects of cinnamaldehyde on human hepatoma cell lines. Food Chem., 2012.

25. Kim, B. H.; Lee, Y. G.; Lee, J.; Lee, J. Y.; Cho, J. Y. Regulatory Effect of Cinnamaldehyde on Monocyte/Macrophage-Mediated Inflammatory Responses. Mediators of Inflammation, 2010.

26. Kim, M. E.; Na, J. Y.; Lee, J. S. Antiinflammatory effects of trans-cinnamaldehyde on lipopolysaccharide-stimulated macrophage activation via MAPKs pathway regulation. Immunopharmacology and Immunotoxicology, 2018.

27. Anand, P.; Murali K.Y.; Tandon, V.; Murthy, P. S.; Chandra, R. Insulinotropic effect of cinnamaldehyde on transcriptional regulation of pyruvate kinase, phosphoenolpyruvate carboxykinase, and GLUT4 translocation in experimental diabetic rats. Chem Biol Interact. Jun 7;186(1):72-81. doi: 10.1016/j.cbi.2010.03.044. 2010.

28. Choi, D. Y.; Baek, Y. H.; Huh, J. E.; et al. Stimulatory effect of Cinnamomum cassia and cinnamic acid on angiogenesis through upregulation of VEGF and Flk-1/KDR expression. Int Immunopharmacology 2009.

29. Yuan, X.; Han, L.; Fu, P.; Zeng, H.; Lv, C.; Chang, W.; Runyon, R. S.; Ishii, M.; Han, L.; Liu,
K.; Fan, T.; Zhang, W.; Liu. R. Cinnamaldehyde accelerates wound healing by promoting angiogenesis via up-regulation of $\mathrm{PI} K \mathrm{~K}$ and MAPK signaling pathways. Laboratory Investigation. https://doi.org/10.1038/s41374018-0025-8, 2017. 